\title{
Manipulator Trajectory Control Combined with Moving Average Filtering Algorithm
}

Jian-Jun Meng

Lanzhou Jiaotong University

Xiao-Tong Chen ( $\nabla$ 565137746@qq.com )

Lanzhou Jiaotong University https://orcid.org/0000-0002-6450-2005

\section{Wen-Zhe Qi}

Lanzhou Jiaotong University

De-Cang Li

Lanzhou Jiaotong University

\section{Ru-Xun Xu}

Lanzhou Jiaotong University

\section{Research Article}

Keywords: Quintic spline interpolation, Mechanical arm, Moving average filtering algorithm, Joint space motion, Forward dynamics

Posted Date: January 13th, 2022

DOI: https://doi.org/10.21203/rs.3.rs-1190663/v1

License: (c) (i) This work is licensed under a Creative Commons Attribution 4.0 International License.

Read Full License 


\section{Manipulator trajectory control combined with moving average filtering algorithm}

Jian-Jun Meng, born in 1966, male, Han, born in Huaiyang, Henan province, is currently an professor at Lanzhou Jiaotong University,China,is doctoral supervisor, research direction: rail transit equipment detection and monitoring technology, etc.

Tel:18435226004 E-mail:565137746@qq.com

Xiao-Tong Chen, born in 1996, female, Han, born in Yangquan, Shanxi Province, is studying for a master's degreeat Lanzhou Jiaotong University,China,. Her research direction is detection technology and automation device.

Wen-Zhe Qi, born in 1966, male, Han, Linxia, Gansu province, is currently an professor at Lanzhou Jiaotong University,China, master tutor, research direction: mechanical and electrical equipment control and monitoring technology, etc.

De-Cang Li, born in 1976, male, Han, born in Huining, Gansu Province, senior engineer, doctor, master supervisor.

Ru-Xun Xu, born in 1986, male, han, male, master, lecturer, research direction: detection technology and automation device, etc.

Corresponding author: Xiao-Tong Chen,E-mail:565137746@qq.com 


\title{
Manipulator trajectory control combined with moving
}

\section{average filtering algorithm}

\author{
Jian-Jun Meng ${ }^{1,2,3} \cdot$ Xiao-Tong Chen ${ }^{1,2,3} \cdot$ Wen-Zhe $\mathrm{Qi}^{2,3} \cdot$ De-Cang Li ${ }^{1,2,3} \cdot \mathrm{Ru}-\mathrm{Xun}$ \\ $\mathrm{Xu}^{1,2,3}$
}

\begin{abstract}
To solve the problem of abnormal angular velocity and angular acceleration in manipulator trajectory motion controlled by quintic spline interpolation algorithm, a manipulator trajectory control algorithm combined with moving average filtering algorithm was proposed. Based on the quintic spline interpolation algorithm, the moving average filtering algorithm was used to clean the abnormal data under the quintic spline interpolation. And the recursive forward dynamics model based on joint space motion was used to design the trajectory motion control of the manipulator. The simulation results show that the manipulator trajectory control algorithm combined with the moving average filtering algorithm has strong constraint ability of diagonal velocity and angular acceleration, and $67 \%$ of the maximum velocity and maximum acceleration of the joint axis of the designed manipulator trajectory are significantly reduced, and the curve is smoother.
\end{abstract}

Keywords: Quintic spline interpolation; Mechanical arm; Moving average filtering algorithm; Joint space motion; Forward dynamics

Xiao-Tong Chen

565137746@qq.com

1 Mechatronics T\&R Institute, Lanzhou Jiaotong University, Lanzhou, 730070, China.

2 School of Mechatronic Engineering, Lanzhou Jiaotong University, Lanzhou, 730070, China.

3 Engineering Technology Center for Informatization of Logistics \& Transport Equipment, Lanzhou, 730070, China.

\section{Introduction}

In the environment of rapid development of intelligence, robotic arms have been developed to a considerable extent in various fields [1-3] due to the advantages of economic manual costs and safety for automated operations. Thus, accurate trajectory planning for robotic arms is particularly important in this case.

The common algorithms for robot arm trajectory motion are joint space motion and Cartesian space motion, etc.To study the trajectory motion of the robot arm for the joint space, the maximum velocity and maximum acceleration of the joint in the motion should be as small as possible, the acceleration at the beginning and end position should be 0 and the angular velocity and angular acceleration should be smoothly over [4.5]. Segmented linear interpolation has good stability and curve convergence, but the smoothness of the curve cannot be guaranteed; Bessel curve [6] does not satisfy the second-order derivative continuity and the interpolation curve does not pass through the path point; B spline interpolation [7] has an interpolation curve that satisfies the second-order derivative continuity but also does not pass through the path point; polynomial interpolation [8] requires certain constraints to be configured in each segment interval, and when the number of nodes is too The calculation is more complicated when the number of nodes is too large, where the acceleration mutation phenomenon exists in the three-sample interpolation, and the five-sample interpolation curve is smooth and does not have the mutation value, but there is still deviation.

In [9], Zhang Liangan et al improved the flexibility and stability of the robotic arm, however, the automation and accuracy of the robotic arm was low, and the footprint was large. Liu Bao and Di Xin [8] reduced the residual vibration of the joint axis using cubic spline trajectory planning, but the pulsation was discontinuous. Li J [10] utilized a spline function and an improved genetic algorithm to solve the problem of generating singular structure points in motion, as well as improved the local optimum problem, but the reliability of the results was poor. A new adaptive control method for a multi-joint flexible robotic arm was proposed by Rahmani B et al. in [11], 
which solved the trajectory tracking problem by using stable inverse control of the robotic arm dynamics. Van Pham C et al. focused on the problem of tracking control manipulator with radial basis function neural network periodic motion and predetermined trajectory of the control dual linkage at joint position, and proposed a robust adaptive control method based on radial basis function neural network in [12]. Generally, some defects exist in the approaches mentioned above, such as the non-smooth motion of the robotic arm, incoherent acceleration trajectories and discontinuous pulses. Therefore, this paper proposes a robotic arm trajectory control algorithm combined with sliding average filtering algorithm, which is used to effectively reduce the joint angular acceleration jumps and achieve smoother angular velocity and angular acceleration curves.

\section{A five spline interpolation}

\section{algorithm combined with a}

\section{sliding average filtering}

\section{algorithm}

\subsection{A five spline interpolation algorithm}

Assuming that the interval $\left[\theta_{0}, \theta_{n}\right]$ is a robotic arm joint node, the definition of the five times spline interpolation is in the interval $\left[\theta_{0}, \theta_{n}\right]$, and the joint split is given as $\theta_{0}<\theta_{1}<\cdots<\theta_{n-1}<\theta_{n} 。 a_{0} 、 a_{1} 、 a_{2} 、 a_{3}$ 、 $a_{4} 、 a_{5}$ are six undetermined coefficients of the quintuple polynomial. The angles, angular velocities and angular accelerations of the beginning and end joints are also constrained, where joint angles satisfy equation (1).

$$
\left\{\begin{array}{c}
\theta(t)=a_{0}+a_{1} t+a_{2} t^{2}+a_{3} t^{3}+a_{4} t^{4}+a_{5} t^{5} \\
\dot{\theta}(t)=a_{1}+2 a_{2} t+3 a_{3} t^{2}+4 a_{4} t^{3}+5 a_{5} t^{4} \\
\ddot{\theta}(t)=2 a_{2}+6 a_{3} t+12 a_{4} t^{2}+20 a_{5} t^{3}
\end{array}\right.
$$

The two adjacent points are then treated as the start and end points of a trajectory, which satisfies equation (2).

$$
\left\{\begin{array}{c}
\theta\left(t_{0}\right)=\theta_{0}=a_{0} \\
\theta\left(t_{f}\right)=\theta_{f}=a_{0}+a_{1} t+a_{2} t^{2}+a_{3} t^{3}+a_{4} t^{4}+a_{5} t^{5} \\
\dot{\theta}\left(t_{0}\right)=\dot{\theta}_{0}=a_{1} \\
\dot{\theta}\left(t_{f}\right)=\dot{\theta}_{f}=a_{1}+2 a_{2} t+3 a_{3} t^{2}+4 a_{4} t^{3}+5 a_{5} t^{4} \\
\ddot{\theta}\left(t_{0}\right)=\ddot{\theta}_{0}=2 a_{2} \\
\ddot{\theta}\left(t_{f}\right)=\ddot{\theta}_{f}=2 a_{2}+6 a_{3} t+12 a_{4} t^{2}+20 a_{5} t^{3}
\end{array}\right.
$$

Equation (3) can be obtained by taking equation (2) into equation (1), which gives the trajectory of the robot arm under the five spline interpolation algorithm.

$$
\left\{\begin{array}{c}
a_{0}=\theta_{0} \\
a_{1}=\dot{\theta}_{0} \\
a_{2}=\ddot{\theta}_{0} / 2 \\
a_{3}=\frac{20 \theta_{f}-20 \theta_{0}-\left(8 \dot{\theta}_{f}+12 \dot{\theta}_{0}\right) t_{f}-\left(3 \ddot{\theta}_{0}-\ddot{\theta}_{f}\right) t_{f}^{2}}{2 t_{f}^{3}} \\
a_{4}=\frac{30 \theta_{f}-30 \theta_{0}-\left(14 \dot{\theta}_{f}+16 \dot{\theta}_{0}\right) t_{f}+\left(3 \ddot{\theta}_{0}-2 \ddot{\theta}_{f}\right) t_{f}^{2}}{2 t_{f}^{4}} \\
a_{5}=\frac{12 \theta_{f}-12 \theta_{0}-\left(6 \dot{\theta}_{f}+6 \dot{\theta}_{0}\right) t_{f}-\left(\ddot{\theta}_{0}-\ddot{\theta}_{f}\right) t_{f}^{2}}{2 t_{f}^{5}}
\end{array}\right.
$$

\subsection{Improved five spline interpolation}

\section{algorithm}

The sliding average filtering algorithm has a strong adaptive capability and excellent suppression of anomalous data. It can obtain the arithmetic average of a set of windowed data, namely the filtered data set, by sliding the window back and forth along the time series.

Set the width of the sliding window to $\mathrm{N}$, if $N=2 k+1$, then

$$
y(n)=\frac{1}{2 k+1} \sum_{i=-k}^{i=k} x(n+i)
$$

where $x(n) 、 y(n)$ represent the input and output respectively.

Although the angular acceleration calculated by the five-spline interpolation algorithm is a smooth curve with no abrupt changes, it still contains the problems of over-sharp values in some regions, absence in homogeneous section, and inability to perform homogeneous planning based on desired angular velocity. In addition, the five-spline interpolation algorithm is one of the polynomial 
interpolation algorithms, with the feature of decelerating the angular acceleration process as the order of the polynomial increases. Therefore, the angles, angular velocities and angular accelerations output from the five-spline interpolation algorithm are used as the data set in the sliding average filter algorithm to obtain smoother data curves by utilizing the advantages of the sliding average filtering algorithm in high smoothness and interference suppression.

\section{Improved robot arm trajectory}

\section{control algorithm}

\subsection{Joint space motion}

The six-degree-of-freedom robot arm is used as an objective, and a motion model for a fixed robot arm is established in the coordinate system $X_{c} Y_{c} Z_{c}$. Set the space vector as $\varphi \in R^{n}$, the end joint node vector as $r_{b} \in R^{n}$, and the two vectors satisfy:

$$
f(\varphi)=r_{b}
$$

where $f(\varphi)$ denotes a differentiable non-linear function.

By transforming the three-dimensional coordinates into two-dimensional coordinates, the position of the end-effector is assumed to be $\left(X_{e}, Y_{e}\right)$, the joint space is assumed to be $\left(\theta_{1}, \theta_{2}\right)$, and the length of each section of the linkage is assumed to be 1 , the position mapping relationship from the joint space to the operation space can be obtained, which is shown in equation (6).

$$
\left\{\begin{array}{c}
X_{\mathrm{e}}=\cos \left(\theta_{1}+\theta_{2}\right)+\cos \theta_{1} \\
Y_{\mathrm{e}}=\sin \left(\theta_{1}+\theta_{2}\right)+\sin \theta_{1}
\end{array}\right.
$$

The structure and parameters of the fixed robotic arm are known, and the $\mathrm{D}-\mathrm{H}$ parameters of the six degree of freedom robotic arm are shown in Table 1.

Table 1:Table of D-H parameters of the six-degree-of-freedom robot arm

\begin{tabular}{ccccc}
\hline$\#$ & $\varphi_{i} / \mathrm{rad}$ & $l_{i} / m$ & $a_{i} / \mathrm{rad}$ & $\alpha_{i} / m$ \\
\hline 1 & $\varphi_{1}$ & 0 & 0 & 0 \\
2 & $\varphi_{2}$ & 0 & 3.20 & 1.57 \\
3 & $\varphi_{3}$ & 0 & 9.75 & 0 \\
4 & $\varphi_{4}$ & 8.87 & 2 & 1.57 \\
5 & $\varphi_{5}$ & 0 & 0 & -1.57 \\
6 & $\varphi_{6}$ & 0 & 0 & 1.57 \\
\hline
\end{tabular}

In the table, $\varphi_{i},{ }^{l_{i}},{ }^{a_{i}}, \alpha_{i}$ represent the joint angle, linkage deflection, linkage length, linkage rotation angle respectively, and the kinematic equation [13] is expressed as equation (7).

$$
r_{b}^{T}=f^{T}(\varphi)=\left[\begin{array}{c}
l_{65}\left(\mathrm{c}_{5} \mathrm{~s}_{32} \mathrm{c}_{1}-s_{5} c_{4} c_{32} c_{1}+s_{5} s_{4} s_{1}\right)+l_{43} s_{32} c_{1}+l_{2} s_{2} c_{1} \\
l_{65}\left(\mathrm{c}_{5} \mathrm{~s}_{32} s_{1}-s_{5} c_{4} c_{32} s_{1}-s_{5} s_{4} s_{1}\right)+l_{43} s_{32} s_{1}+l_{2} s_{2} s_{1} \\
l_{65}\left(s_{5} c_{4} \mathrm{~s}_{32}+c_{5} c_{32}\right)+l_{43} c_{32}+l_{2} c_{2}+l_{1}+l_{0}
\end{array}\right]^{T}
$$

Where $l_{i}$ means the $\mathrm{i}$-th linkage length, $l_{65}$ means $l_{6}+l_{5}, l_{43}=l_{4}+l_{3}, c_{i}$ means $\cos \varphi_{i}(i=1,2, \cdots, 6) \quad, \quad s_{i} \quad$ means $\sin \varphi_{i}(i=1,2, \cdots, 6), s_{32}$ means $\sin \left(\varphi_{3}+\varphi_{2}\right)$, and $c_{32}$ means $\cos \left(\varphi_{3}+\varphi_{2}\right)$.

\subsection{Improvement of joint space motion of robotic arm}

The joint axes are biased during the motion of the robot arm and the angles, angular velocity and angular acceleration [14] datasets are generated. Then, the datasets of angle, angular velocity and angular acceleration are cleaned using the sliding average filtering algorithm described above.

Assuming the angle and angular velocitie of the robotic arm joints is $(\theta, \dot{\theta})$. The joint moment $\tau$ is calculated from the joint acceleration $\ddot{\theta}$, which can also be used to address the joint acceleration for the next stage of motion by forward dynamics [15-17]. The algorithm for calculating the forward dynamics of the system consists of three processes: forward recursion, reverse recursion, and again forward recursion. These processes traverse all connecting rods in turn according to the principle of depth-first, reverse depth-first, and the depth-first [18]. Equations (8), (9) and (10) are the formulas for the calculation of Forward Kinematics.

$$
\left\{\begin{array}{c}
\mathrm{T}_{\lambda(\mathrm{i}), \mathrm{i}}=\mathrm{f}(\theta) \\
\mathrm{V}_{\mathrm{i}}=\mathrm{Ad}_{\mathrm{T}_{\lambda(\mathrm{i}), \mathrm{i}}^{-1}} \mathrm{~V}_{\lambda(\mathrm{i})}+\mathrm{S}_{\mathrm{i}} \dot{\theta}_{\mathrm{i}} \\
\eta_{\mathrm{i}}=\operatorname{ad}_{\mathrm{V}_{\mathrm{i}}} \mathrm{S}_{\mathrm{i}} \dot{\theta}_{\mathrm{i}}+\dot{\mathrm{S}}_{\mathrm{i}} \dot{\theta}_{\mathrm{i}}
\end{array}\right.
$$




$$
\begin{aligned}
& \hat{\mathrm{I}}_{\mathrm{i}}=\mathrm{I}_{\mathrm{i}}+\sum_{\mathrm{k} \in \mu(\mathrm{i})} \operatorname{Ad}_{\mathrm{T}_{\mathrm{i}, \mathrm{k}}^{-1}}^{*} \prod_{\mathrm{k}} \operatorname{Ad}_{\mathrm{T}_{\mathrm{i}, \mathrm{l}}^{-1}} \\
& \hat{B}_{i}=-\mathrm{ad}_{\mathrm{v}_{\mathrm{i}}}^{*} \mathrm{I}_{\mathrm{i}} \mathrm{V}_{\mathrm{i}}-\mathrm{F}_{\mathrm{i}}^{\mathrm{ext}}+\sum_{\mathrm{k} \in \mu(\mathrm{i})} \operatorname{Ad}_{\mathrm{T}_{\mathrm{i}, \mathrm{k}}}^{*} \beta_{\mathrm{k}} \\
& \Psi_{\mathrm{i}}=\left(\mathrm{S}_{\mathrm{i}}^{\mathrm{T}} \hat{\mathrm{I}}_{\mathrm{i}} \mathrm{S}_{\mathrm{i}}\right)^{-1} \\
& \prod_{i}=\hat{I}_{i}-\hat{I}_{i} S_{i} \Psi_{i} S_{i}^{T} \hat{I}_{i} \\
& \beta_{\mathrm{i}}=\hat{B}_{\mathrm{i}}+\hat{\mathrm{I}}_{\mathrm{i}}\left\{\eta_{\mathrm{i}}+\mathrm{S}_{\mathrm{i}} \Psi_{\mathrm{i}}\left[\tau_{\mathrm{i}}-\mathrm{S}_{\mathrm{i}}^{\mathrm{T}}\left(\hat{\mathrm{I}}_{\mathrm{i}} \eta_{\mathrm{i}}+\hat{B}_{\mathrm{i}}\right)\right]\right\} \\
& \left\{\begin{array}{c}
\ddot{\theta}_{\mathrm{i}}=\Psi_{\mathrm{i}}\left[\tau_{\mathrm{i}}-\mathrm{S}_{\mathrm{i}}^{\mathrm{T}} \hat{\mathrm{I}}_{\mathrm{i}}\left(\mathrm{Ad}_{\mathrm{T}_{\lambda(\mathrm{i}) \mathrm{i}}^{-1}} \dot{\mathrm{V}}_{\lambda(\mathrm{i})}+\eta_{\mathrm{i}}\right)-\mathrm{S}_{\mathrm{i}}^{\mathrm{T}} \hat{\mathrm{B}}_{\mathrm{i}}\right] \\
\dot{\mathrm{V}}_{\mathrm{i}}=\mathrm{Ad}_{\mathrm{T}_{\lambda(\mathrm{i}), \mathrm{i}}^{-1}} \dot{\mathrm{V}}_{\lambda(\mathrm{i})}+\mathrm{S}_{\mathrm{i}} \ddot{\theta}_{\mathrm{i}}+\eta_{\mathrm{i}} \\
\mathrm{F}_{\mathrm{i}}=\hat{\mathrm{I}}_{\mathrm{i}} \dot{\mathrm{V}}_{\mathrm{i}}+\hat{\mathrm{B}}_{\mathrm{i}}
\end{array}\right.
\end{aligned}
$$

Where denotes the i-th joint, denotes the i-th joint parent, denotes the i-th shutdown sub-joint, denotes the coordinate transformation matrix, denotes the generalized inertia of the i-th joint, denotes the inertia matrix of the $\mathrm{i}$-th joint and at each end body, denotes the generalized velocity of the $\mathrm{i}$-th joint in the solid joint coordinate system, denotes the induced partial force, denotes the i-th joint generalized force, denoted as the correlation case at the i-th joint, $d$ denoted as the disturbance moment vector, denoted as the deviation of the real dynamics model of the robot arm from the nominal dynamics model, denoted as the dyadic operator, denoted as the $n * n$ antisymmetric matrix of the i-th joint, denoted as the bias force of the i-th joint, denoted as the known part of the generalized force.

\section{Experimental simulation and result analysis}

\subsection{Sliding average filtering amplitude and} frequency response simulation and analysis

Assuming that the input signal is a continuous function,

$$
y(t)=\frac{1}{T_{0}} \int_{t-T_{0}}^{t} f(x) d x
$$

Where $f(x)$ and $y(t)$ is the input and output respectively, and $T_{0}$ is the length of sliding filter.

For the sliding average filter amplitude-frequency response, assuming the filter period is $T_{0}$, and the input signal is

$$
\begin{gathered}
f(x)=\cos \left(\frac{2 \pi}{T} x\right) \\
T_{0}=k T+R,(k=0,1,2, \cdots ; 0 \leq R<T)
\end{gathered}
$$

Taking equations (12) and (13) into equation (11) and calculating the amplitude of the input signal as

$$
\begin{aligned}
& A=\frac{2}{T_{0}} \int_{0}^{R / 2} \cos \left(\frac{2 \pi}{T} x\right) d x \\
& A=\frac{T}{n T_{0}} \sin \left(\frac{\pi T_{0}}{T}-k \pi\right)
\end{aligned}
$$

The response of the sliding average filtered amplitude and frequency is shown in Figure 1. It can be seen that the continuous functions are smoothed and filtered. And the non-smooth data are averaged locally in some way and considered as close to smooth on a suitable small interval to reduce the random ups and downs caused by errors.

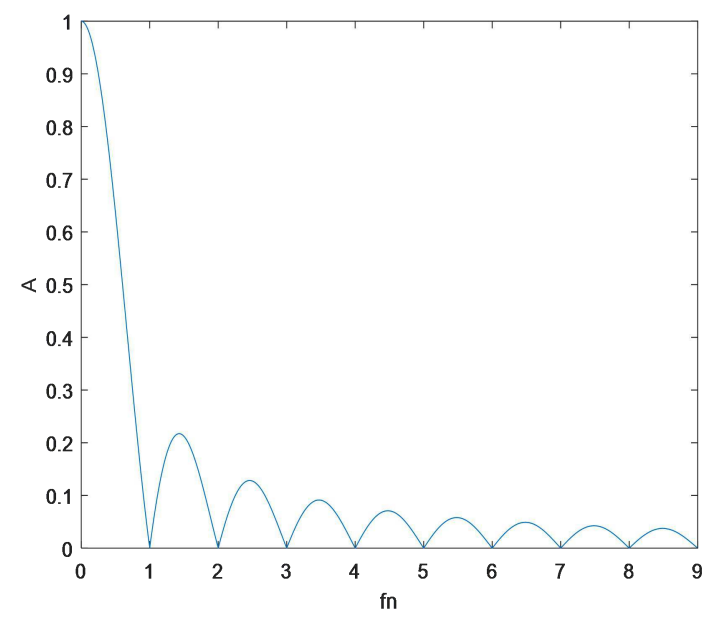

Figure 1:Sliding average filtering amplitude and frequency response

\subsection{Simulation results and analysis of five} times spline interpolation algorithm before and after improvement

The five spline interpolation algorithm and the five spline interpolation algorithm combined with the sliding average filtering algorithm are simulated by MATLAB and the simulation results are shown in Figure 2. 

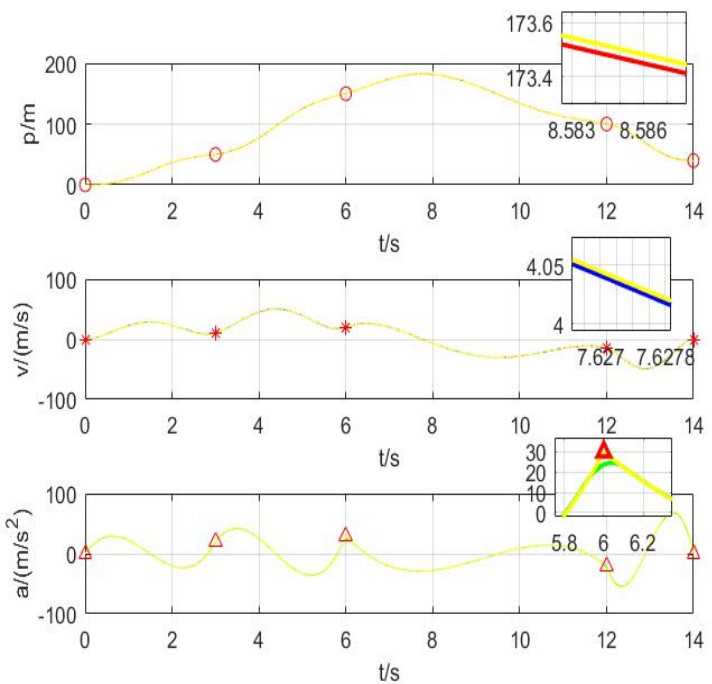

(a) Simulation comparison

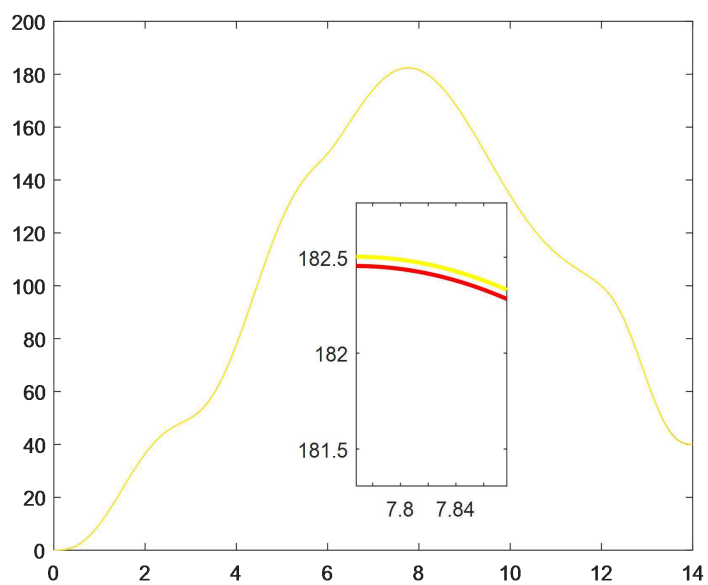

(b) Location comparison

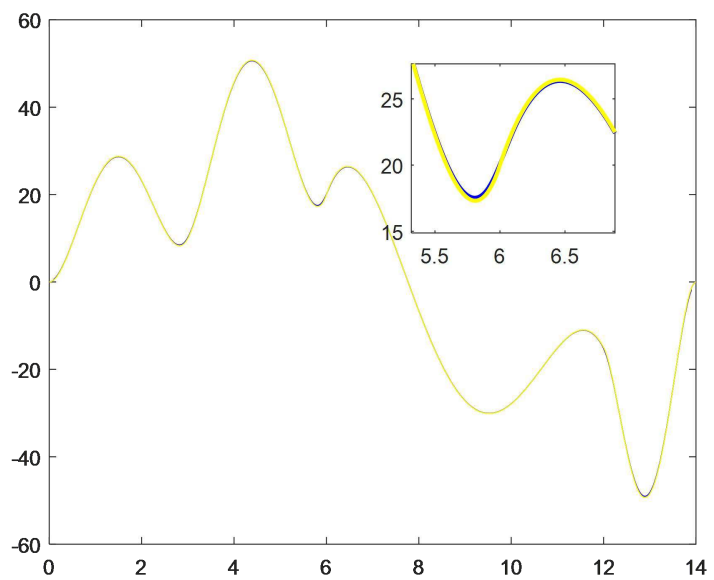

(c) Speed comparison

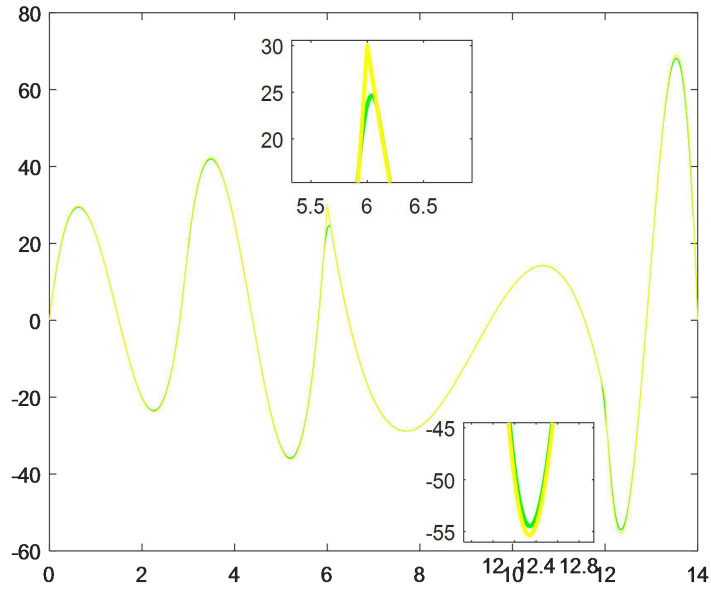

(d) Acceleration comparison

Figure 2:Comparison of five spline interpolation algorithm before and after improvement

Where the yellow curve indicates the five spline interpolation algorithm simulation curve; red, blue and green indicate the five spline interpolation algorithm combined with the sliding average filtering algorithm to compare position, velocity and acceleration respectively. Figure 2(a) is a general view of the five spline interpolation algorithm and the five spline interpolation algorithm combined with the sliding average filtering algorithm comparing position (Fig. 2(b)), velocity (Fig. 2(c)) and acceleration (Fig. 2(d)). As can be seen from the enlarged diagram, the five spline interpolation algorithm combined with the sliding average filtering algorithm effectively improves the smoothness of the curve, especially in the case of acceleration. Therefore, the problem of generating anomalous values in the five spline interpolation algorithm is effectively solved by combining the sliding average filter algorithm with the five spline interpolation algorithm.

\subsection{Simulation results and analysis of joint space motion of robot arm before and after improvement}

According to the simulation and analysis of the five times spline interpolation algorithm before and after the above improvement, it can be seen that the advantages of the five times spline interpolation algorithm combined with the sliding average filtering algorithm to clean the data are now applied to the joint space motion of the robot arm, and the simulation 
results of the joint space motion before and after the improvement are shown in Figure 3.
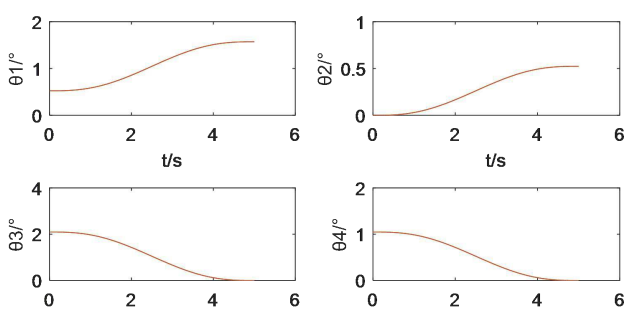

$\mathrm{t} / \mathrm{s}$
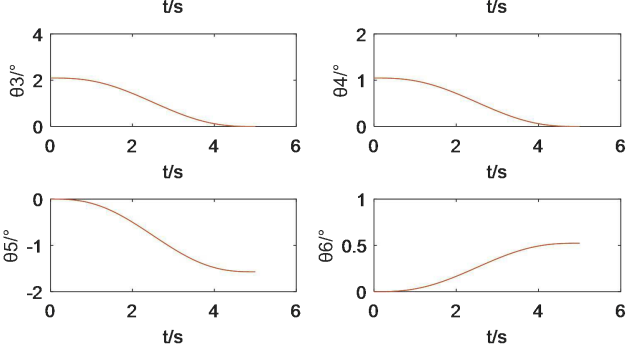

(a) Angular comparison
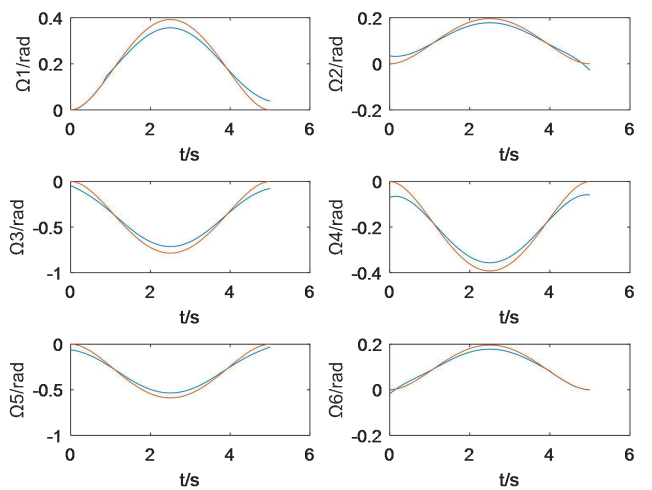

(b) Angular velocity comparison

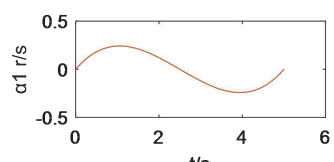

$\mathrm{t} / \mathrm{s}$
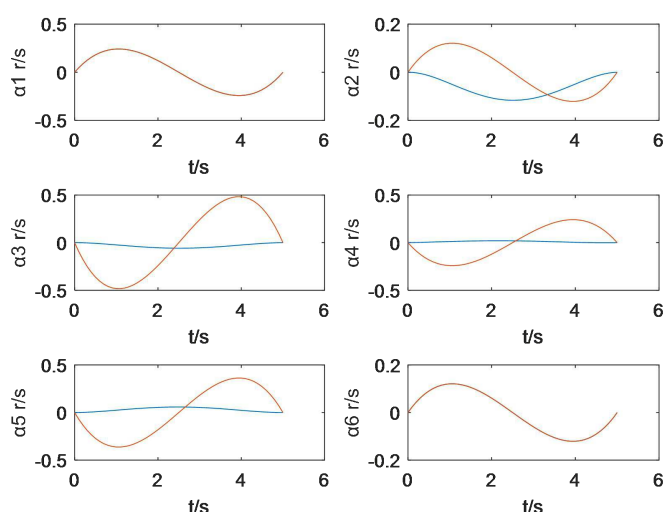

(c) Comparison of angular acceleration

Figure 3:Comparison of joint space motion of the arm before and after improvement

Among them, the red line is the joint space motion simulation result of the robotic arm, and the blue line is the joint space motion simulation result of the robotic arm after the improvement. From Fig.3(a), it can be seen that the end joint actuator motion angles before and after the improvement overlap, which indicates that the robotic arm trajectory control algorithm combined with the sliding average filter algorithm and the original trajectory control algorithm of the robotic arm are calculated for the same robotic arm trajectory motion; from Fig.3(b), it can be seen that $67 \%$ of the maximum velocity of the joint axis in the motion of the improved robotic arm is significantly reduced and the curve is smooth; from Fig.3(c), it can be seen that The angular acceleration of the improved robotic arm is significantly reduced, which indicates that the robotic arm trajectory control algorithm combined with the sliding average filtering algorithm effectively solves the problems of the robotic arm motion that is not smooth and the acceleration is abruptly changed.

Table 2:Comparison of angular velocity before and after improvement

\begin{tabular}{|c|c|c|c|c|c|c|c|c|c|c|c|c|}
\hline \multirow{2}{*}{ \# } & \multicolumn{2}{|c|}{$\Omega_{1}$} & \multicolumn{2}{|c|}{$\Omega_{2}$} & \multicolumn{2}{|c|}{$\Omega_{3}$} & \multicolumn{2}{|c|}{$\Omega_{4}$} & \multicolumn{2}{|c|}{$\Omega_{5}$} & \multicolumn{2}{|c|}{$\Omega_{6}$} \\
\hline & Tra & $\operatorname{Im}$ & Tra & $\operatorname{Im}$ & Tra & $\operatorname{Im}$ & Tra & $\operatorname{Im}$ & Tra & $\operatorname{Im}$ & Tra & Im \\
\hline Min. & \multirow{2}{*}{0} & \multirow{2}{*}{0} & \multirow{2}{*}{0} & -0.02 & -0.78 & -0.71 & -0.39 & -0.35 & -0.58 & -0.53 & \multirow{2}{*}{0} & -0.016 \\
\hline value & & & & 729 & 54 & 34 & 27 & 66 & 9 & 49 & & 99 \\
\hline Maximum & 0.392 & 0.356 & 0.196 & 0.178 & \multirow{2}{*}{0} & -0.04 & \multirow{2}{*}{0} & -0.05 & \multirow{2}{*}{0} & -0.03 & 0.19 & \multirow{2}{*}{0.1783} \\
\hline value & 7 & 6 & 3 & 3 & & 53 & & 819 & & 14 & 63 & \\
\hline \multirow{2}{*}{ \# } & \multicolumn{2}{|c|}{$\alpha_{1}$} & \multicolumn{2}{|c|}{$\alpha_{2}$} & \multicolumn{2}{|c|}{$\alpha_{3}$} & \multicolumn{2}{|c|}{$\alpha_{4}$} & \multicolumn{2}{|c|}{$\alpha_{5}$} & \multicolumn{2}{|c|}{$\alpha_{6}$} \\
\hline & Tra & $\mathrm{Im}$ & Tra & $\mathrm{Im}$ & Tra & $\mathrm{Im}$ & Tra & $\mathrm{Im}$ & Tra & $\mathrm{Im}$ & Tra & Im \\
\hline
\end{tabular}




\begin{tabular}{ccccccccccccc}
\hline Min. & -0.24 & -0.24 & -0.12 & -0.11 & -0.48 & -0.05 & -0.24 & -0.001 & -0.36 & -2.07 & -0.12 & -0.120 \\
value & 15 & 15 & 08 & 65 & 3 & 823 & 15 & 179 & 23 & $9 \mathrm{e}-16$ & 08 & 8 \\
Maximum & 0.241 & 0.241 & 0.12 & 1.507 & & -4.01 & 0.241 & 0.020 & 0.362 & 0.058 & 0.120 & 0.120 \\
value & 5 & 5 & 08 & $\mathrm{e}-16$ & & $2 \mathrm{e}-17$ & 5 & 14 & 3 & 23 & 8 & 8 \\
\hline
\end{tabular}

Note: Tra means Traditional, Im means Improvements. 
Table 2 and Table 3 are the comparison data table of angular velocity before and after the improvement and the comparison data table of angular acceleration before and after the improvement, involving the minimum and maximum values of angular velocity and angular acceleration of six joints. According to the data in Table 2, it can be seen that the minimum value of angular velocity slightly increases and the maximum value generally decreases, for example, the minimum value remains unchanged and the maximum value decreases by 0.0361 , overall, the minimum value of angular velocity under the improved algorithm is $5.32 \%$ higher than that under the traditional algorithm, and the maximum value of angular velocity under the improved algorithm is $6.84 \%$ lower than that under the traditional algorithm. According to the data in Table 3, it can be seen that the angular acceleration also has a slight increase in the minimum value and a general decrease in the maximum value, with an increase of $48.5 \%$ in the minimum value and a decrease of $62.4 \%$ in the maximum value.

\section{Conclusion}

A robotic arm trajectory control algorithm that uses a six degree of freedom robotic arm and combines a sliding average filtering algorithm is proposed to effectively reduce the maximum velocity and maximum acceleration of the end joint motion.

The results show that the five times spline interpolation algorithm is effective in improving the position, velocity and acceleration curves, however, the inflection points of some curves are still sharp. While the five-spline interpolation algorithm combined with the sliding average filtering algorithm could optimize the anomalous inflection points in the curve without changing the original position, velocity and acceleration curves, and obtain a smoother curve.

The comparison of the robot arm joint spatial motion before and after the improvement illustrate that robotic arm trajectory control algorithm combined with sliding average filtering algorithm achieves the requirement of spatial trajectory motion of robotic arm joints. There were $67 \%$ of joints with significantly reduced maximum velocities, lower angular accelerations and smooth curves during the movement.

The results show that the arm trajectory control algorithm combined with the sliding average filtering algorithm provides a smoother trajectory motion with less error than the original arm trajectory control algorithm, and the maximum velocity and maximum acceleration of the joint are significantly reduced, which protects the arm control system to a certain extent.

\section{Availability of data and materials}

The code is referenced and modified on https://blog.csdn.net/jldemanman/article/details/79303 021、https://blog.csdn.net/weixin_44947987/article/det ails/103843155

\section{Competing Interest}

No potential conflict of interest was reported by the authors.

\section{Funding}

National Natural Science Foundation of China (62063013) (72061021); Gansu Provincial Science and Technology Program (20JR10RA251, 21JR7RA284), Lanzhou Jiaotong University Youth Fund Project (20210018).

\section{Authors' contributions}

Corresponding author Xiao-Tong Chen ensured that all listed authors had approved the manuscript, 
including the authors' names and order, and that all authors had received the submission and all substantive correspondence with the editor, as well as complete comments verifying that all data, graphics, and code met the field's and journal's standards for transparency and reproducibility.Each author is expected to have made substantial contributions to the conception OR design of the work; OR the acquisition, analysis, OR interpretation of data; OR the creation of new software used in the work.

\section{Acknowledgements}

Thanks to other teachers for their pointers on writing and to CSDN, a site with viable code support.

\section{References}

[1] Wang Youyu,Tang Zixin,Li Delun,et al. Space robotic arm accuracy analysis research and software implementation[J]. Manned Spaceflight,2021,27(01): 59-65.

[2] Zhao Yunlong,Che Renfei,Chen Jiahui. Intelligent phase change strategy for distribution networks based on differential evolutionary algorithm[J]. Journal of Shandong University (Engineering Edition), 2021, 51(1), DOI:10.6040 /j.issn.1672-3961.0.2020.110.

[3] Xi Houyin,Zhang Dong,Zhou Tao,et al. Research on the method of identifying and grasping overlapping tomato fruits by picking robot[J]. Agricultural mechanization research,2021,43(12),17-23+50.

[4] Du Yanfeng, Wang Cong. Residual vibration control of flexible robotic arm[J]. Vibration and shock,2019,38(7):165-171.

[5] Fang Y, Hu J, Shao Q Q, et al. Fifth order trajectory planning for reducing residual vibration[C]// Proceedings of 2019 International Conference on Advanced Robotics and Mechatronics. toyonaka, Japan: IEEE, 2019: 999-1004.

[6] Huang Q H, Huang Y P, Hu W, et al. Bezier Interpolation for 3-D Freehand Ultrasound[J]. IEEE Transactions on Human-Machine Systems, 2015, 45(3): 385-392.

[7] He XM, Shi GJ, Wu MP, et al. Application of B-sample to screw rotor profile inverse design method species[J]. Mechanical Science and Technology, 2018,37(1):43-54.

[8] Liu B, Di X, Han LH. Application of improved cubic spline interpolation in robotic arm trajectory planning. Mechanical Science and Technology $[\mathrm{J} / \quad$ OL]. (2020-01-21) [2020-09-12].

https://kns.cnki.net/kcms/detail/11.2127.TP.20201023.1737. 011.html.

[9] Zhang L.A., Ma Y.D., Shan J.Z., et al. Optimal design of 4-degree-of-freedom palletizing robot with local closed-chain dynamics $[\mathrm{J}]$. Journal of Agricultural Engineering.2013,44(11):336-341

[10] Li J,Shu ZB,Wang SZ. Robot trajectory planning based on spline function and improved genetic algorithm[J]. Manufacturing Technology and Machine Tools,2017(7):91-95.

[11] Rahmani B , Belkheiri M . Adaptive Neural Network Output Feedback Control for Flexible Multi-Link Robotic Manipulators[J]. International Journal of Control, 2018:1-35.

[12] Van Pham C , Wang Y N . Robust Adaptive Trajectory Tracking Sliding mode control based on Neural networks for Cleaning and Detecting Robot Manipulators[J]. Journal of Intelligent \& Robotic Systems, 2015, 79(1):101-114.

[13] Xiao, Lin, Zhou, Wenhui. Design and verification of coordinated motion scheme for mobile robotic $\operatorname{arm}[\mathrm{J}]$. Journal of Zhongshan University (Natural Science Edition).2016,55(2):52-57.

[14] Zhou Yuan. Computer simulation planning of Cartesian spatial trajectory of robot $\operatorname{arm}[\mathrm{J}]$. Tool Technology.2016,50(9):82-86.

[15] Liu P,Wang Q,Zhang W. Robotic arm dynamics modeling and control simulation[J]. Automation and Instrumentation,2017(03):9-12.

[16] Shao B,Wu H,Cheng S,et al. Dynamics and control of active-passive joint robot based on Lie group Lie algebra[J]. China Mechanical Engineering,2010(3):253-257.

[17] Shao B. Recursive dynamics of unified open and closed-loop mechanical multibody systems based on Lie group Lie algebra[D]. Nanjing: Nanjing University of Aeronautics and Astronautics, 2010.

[18] Bai Chengchao, Yan Zhuo, Song Junlin. Combined deep learning for visual grasping control of robotic $\operatorname{arm}[\mathrm{J}]$. Manned Space.2018,24(3):299-307.

\section{Biographical notes}

Jian-Jun Meng, born in 1966, male, Han, born in Huaiyang, 
Henan province,is currently an professor at Lanzhou Jiaotong University,China,is doctoral supervisor, research direction: rail transit equipment detection and monitoring technology, etc.

\section{Tel:18435226004 E-mail:565137746@qq.com}

Xiao-Tong Chen, born in 1996, female, Han, born in Yangquan,

Shanxi Province, is studying for a master's degreeat Lanzhou

Jiaotong University,China,. Her research direction is detection technology and automation device.

Wen-Zhe Qi, born in 1966, male, Han, Linxia, Gansu province, is currently an professor at Lanzhou Jiaotong University,China, master tutor, research direction: mechanical and electrical equipment control and monitoring technology, etc.

De-Cang Li, born in 1976, male, Han, born in Huining, Gansu Province, senior engineer, doctor, master supervisor.

Ru-Xun Xu, born in 1986, male, han, male, master, lecturer, research direction: detection technology and automation device, etc. 